\title{
Neutron stars in a class of nonlinear relativistic models
}

\author{
A. R. Taurines, ${ }^{1}$ C. A. Z. Vasconcellos, ${ }^{1}$ M. Malheiro, ${ }^{2}$ and M. Chiapparini ${ }^{3}$ \\ ${ }^{1}$ Instituto de Física, Universidade Federal do Rio Grande do Sul, Porto Alegre 91501-970, Brazil \\ ${ }^{2}$ Instituto de Física, Universidade Federal Fluminense, Niterói 24210-340, Brazil \\ ${ }^{3}$ Departamento de Física Teórica, Universidade do Estado do Rio de Janeiro, Rio de Janeiro 20559-900, Brazil
}

(Received 29 September 2000; revised manuscript received 7 February 2001; published 1 May 2001)

\begin{abstract}
In this work we introduce a class of relativistic models for nuclear matter and neutron stars which exhibits a parametrization, through mathematical constants, of the nonlinear meson-baryon couplings. For appropriate choices of the parameters, it recovers current quantum hadrodynamics models found in the literature: the Walecka model and Zimanyi-Moszkowski models (ZM and ZM3). For other choices of parameters, the models give very interesting and new physical results. The phenomenology of neutron stars in ZM models is presented and compared to the phenomenology obtained in other versions of the Walecka model. We have found that the ZM3 model is too soft, and predicts a very small maximum neutron star mass, $\sim 0.72 M_{\odot}$. A strong similarity between the results of ZM-like models and those with exponential couplings is noted. The sensibility of the results to the specific choice of the values for the binding energy and saturation density is pointed out. Finally, we discuss the very intense scalar condensates found in the interior of neutron stars, which may lead to negative effective masses.
\end{abstract}

DOI: 10.1103/PhysRevC.63.065801

PACS number(s): 26.60.+c, 21.65.+f, 95.30.Cq, 97.60.Jd

\section{INTRODUCTION}

Since the discovery of the first pulsar in 1968 [1] and its identification as a rotating neutron star, the structure, composition, dynamics, and evolution of these astrophysical objects became important themes of theoretical and phenomenological research. According to an early suggestion [2], neutron stars evolve from an initially hot protoneutron star that forms in the collapse of a massive star in the supernova phenomenon. At densities exceeding that of nuclear matter, important static properties of a neutron star, such as the mass-radius relation, the crust extent, the distribution of the stellar moments of inertia, and the central density, may be determined by its equation of state (EOS) [3].

During this period, there was a continuous enhancement concerning relativistic microscopic calculations of the EOS of neutron stars, improving our understanding of the structure of these stellar objects. In particular, any theory must at least account for a neutron star as massive as the most massive observed pulsar; the knowledge of pulsar masses provides a very important constraint on the theory.

More recent calculations based on relativistic properties of nuclear matter at high densities indicate that the equations of state are considerable stiffer than those predicted by nonrelativistic approaches. As a result, the mass of a neutron star is believed to be at least as large as $(1.6-2.1) M_{\odot}$.

From the theoretical point of view, quantum chromodynamics $(\mathrm{QCD})$ represents the most profound description of the strong interaction and would be the ideal tool for neutron star applications. However, the highly nonlinear behavior of QCD at the hadronic energy scales inhibits any practical calculations leading most theorists to search for phenomenological descriptions of the structure of nuclear matter. One of these alternative approaches is quantum hadrodynamics (QHD) [4], a relativistic quantum field theory based on a local Lagrangian density which uses baryon and meson fields as the relevant degrees of freedom. This model provides a consistent theoretical framework for describing such a relativistic interacting many-body system and, based on it, Glendenning presented [5] a very comprehensive treatment of the matter in neutron stars, using an extended version which included leptons and the fundamental baryonic octet.

Alternative versions of the Walecka model, namely the Boguta-Bodmer (BB) [6] and Zimanyi-Moszkowski (ZM) models $[7,8]$, were developed to improve the description of the nucleon effective mass $M^{\star}$ (too low) and compression modulus of nuclear matter $K$ (too high) as attained with the original approach. Boguta and Bodmer introduced cubic and quartic scalar self-interactions in the Lagrangian while the ZM models heightened the delineation of these quantities by replacing the Yukawa scalar coupling term by a derivative coupling contribution. This derivative coupling may be interpreted alternatively as a phenomenological coupling between the scalar neutral meson and the nucleon fields through the introduction of a baryon density dependence in the scalar, vector, and isovector coupling constants of the theory $[9,10]$.

In this work we analyze the structure of neutron stars by introducing a QHD Lagrangian with a parametrized meson coupling contribution [11]. This phenomenological approach contains high-order self-coupling contributions of the meson fields, and permits one, in particular, to restore the results obtained with Walecka, ZM, and ZM3 models by making suitable choices of the values of the mathematical parameters of the theory. The control of the analytical form of the couplings allows us to investigate other values of these parameters which give new physical results. By extending the formalism to include hyperons and leptons, we investigate several static bulk properties of neutron stars using the Walecka, ZM, and ZM3 models, the latter two being applied to this problem for the first time. As we have a class of models, we are able to relate nuclear matter and neutron star properties. In particular, we have found that some of the studied models describe very strong scalar fields in the interior of the neutron stars, leading to a negative nucleon effective mass. 


\section{GENERAL CHARACTERISTICS OF NEUTRON STAR MATTER}

In the evolution of the protoneutron star, many different reactions can occur. The electric charge and baryon number are conserved on a long-time scale in comparison to the lifetime of the star. In the core of the protoneutron star, the Fermi energy of the nucleons exceed the hyperon masses and these particles can be produced in strong interaction processes with conservation of strangeness in reactions such that

$$
n+n \rightarrow n+\Lambda+K
$$

However, strangeness is not conserved on the time scale of the star, since there occurs a diffusion of neutrinos and photons to the surface of the star: processes like

$$
K^{0} \rightarrow 2 \gamma, \quad K^{-} \rightarrow \mu^{-}+\bar{\nu}_{\mu}, \ldots
$$

can no longer be reversed, and a net strangeness appears.

In this evolution process, the star reaches chemical equilibrium, a degenerate state where, from the point of view of its hadronic and leptonic composition, further reactions are not possible. As an example, in an ideal degenerate system of protons, neutrons, and electrons at chemical equilibrium, particle levels are filled in such a way that neutron beta decay or proton inverse beta decay are not energetically favored.

In general, if one takes into account the fundamental baryon octet and lepton degrees of freedom (electrons and muons), the following chemical equilibrium equation then holds (see the Appendix):

$$
\mu_{i}=q_{b, i} \mu_{n}-q_{e, i} \mu_{e},
$$

where $i=p, n, \Lambda, \Sigma^{0}, \Sigma^{-}, \Sigma^{+}, \Xi^{-}, \Xi^{0}, e, \mu ; q_{b, i}$ represents the baryon number, and $q_{e, i}$ the electric charge of species $i$. In this way, the conditions for $\beta$ equilibrium can be summarized as

$$
\begin{gathered}
\mu_{\Sigma^{0}}=\mu_{\Xi^{0}}=\mu_{\Lambda}=\mu_{n}, \\
\mu_{\Sigma^{-}}=\mu_{\Xi^{-}}=\mu_{n}+\mu_{e}, \\
\mu_{\Sigma^{+}}=\mu_{p}=\mu_{n}-\mu_{e} .
\end{gathered}
$$

Additionally to these chemical equilibrium conditions, we have to take into account that a neutron star is electrically neutral $[5,12]$. This easily follows from the balance between the repulsive electrical and the attractive gravitational forces.

\section{BOGUTA-BODMER MODEL}

\section{A. Theory}

In this section we study baryon and lepton populations in neutron stars by using the Boguta-Bodmer model with hyperon degrees of freedom. In spite of this study having already been done [5], we reproduce its main results as a guide for the development in Sec. IV of our new class of nonlinear relativistic models.
The BB model describes the complex fermionic composition of neutron stars as a generalization of the $\sigma, \omega$, and $\varrho$ theory:

$$
\begin{aligned}
\mathcal{L}= & \sum_{B} \bar{\psi}_{B}\left[i \gamma_{\mu} \partial^{\mu}-\left(M_{B}-g_{\sigma B} \sigma\right)-g_{\omega B} \gamma_{\mu} \omega^{\mu}\right. \\
& \left.-\frac{1}{2} g_{\varrho B} \gamma_{\mu} \tau \cdot \varrho^{\mu}\right] \psi_{B}+\sum_{l} \bar{\psi}_{l}\left(i \gamma_{\mu} \partial^{\mu}-m_{l}\right) \psi_{l} \\
& +\frac{1}{2}\left(\partial_{\mu} \sigma \partial^{\mu} \sigma-m_{\sigma}^{2} \sigma^{2}\right)-\frac{1}{4} \omega_{\mu \nu} \omega^{\mu \nu}+\frac{1}{2} m_{\omega}^{2} \omega_{\mu} \omega^{\mu} \\
& -\frac{1}{4} \varrho_{\mu \nu} \cdot \varrho^{\mu \nu}+\frac{1}{2} m_{\varrho}^{2} \varrho_{\mu} \cdot \varrho^{\mu}-\frac{1}{3} b M\left(g_{\sigma} \sigma\right)^{3} \\
& -\frac{1}{4} c\left(g_{\sigma} \sigma\right)^{4} .
\end{aligned}
$$

This Lagrangian density describes a system of eight baryons ( $B=p, n, \Lambda, \Sigma^{-}, \Sigma^{0}, \Sigma^{+}, \Xi^{-}, \Xi^{0}$ ) coupled to three mesons $(\sigma, \omega, \varrho)$ and two free lepton species $\left(l=e^{-}, \mu^{-}\right)$. The scalar and vector coupling constants in the theory, $g_{(\sigma, \omega)}$, and the coefficients $b$ and $c$ are determined to reproduce, at saturation density $\rho_{0}=0.15 \mathrm{fm}^{-3}$, the binding energy of nuclear matter, $B=-16 \mathrm{MeV}$, the compression modulus of nuclear matter, $K=250 \mathrm{MeV}$, and the nucleon effective mass, $M^{\star} / M=0.75$. In fact, the values for these two last quantities are not well established, and we have just taken the most used values in the literature of the BB model. Additionally, to describe the symmetry energy coefficient, $a_{4}$ $=32.5 \mathrm{MeV}$, we determine the isovector coupling constant $g_{\varrho}$. We have found

$$
\begin{gathered}
\left(\frac{g_{\sigma}}{m_{\sigma}}\right)^{2}=9.86 \mathrm{fm}^{2}, \quad\left(\frac{g_{\omega}}{m_{\omega}}\right)^{2}=5.85 \mathrm{fm}^{2}, \\
\left(\frac{g_{\varrho}}{m_{\varrho}}\right)^{2}=4.80 \mathrm{fm}^{2}, \quad b=0.00103, \quad c=0.0100 .
\end{gathered}
$$

In comparison with the results obtained in Ref. [5], one should recall that this author fitted the coupling constants of the theory with $B=-15.95 \mathrm{MeV}, \rho_{0}=0.145 \mathrm{fm}^{-3}, K$ $=285 \mathrm{MeV}, M^{\star} / M=0.77$, and $a_{4}=36.8 \mathrm{MeV}$.

Using the Euler-Lagrange equations, the Dirac equation for uniform matter, in a momentum representation, is

$$
\left[\gamma_{\mu}\left(k^{\mu}-g_{\omega B} \omega^{\mu}-\frac{1}{2} g_{\varrho B} \boldsymbol{\tau} \cdot \varrho^{\mu}\right)-M_{B}^{*}(\sigma)\right] \psi_{B}(k)=0,
$$

where $M_{B}^{*}(\sigma) \equiv M_{B}-g_{\sigma B} \sigma$ is the effective mass of the baryonic species $B$. Furthermore, by applying the mean-field approximation, the $\omega_{0}, \varrho_{03}$, and $\sigma$ meson field equations for uniform static matter are

$$
g_{\omega} \omega_{0}=\left(\frac{g_{\omega}}{m_{\omega}}\right)^{2} \sum_{B} \chi_{\omega, B} \rho_{B},
$$




$$
\begin{gathered}
g_{\varrho} \varrho_{03}=\left(\frac{g_{\varrho}}{m_{\varrho}}\right)^{2} \sum_{B} \chi_{\varrho, B} I_{3 B} \rho_{B}, \\
g_{\sigma} \sigma=\left(\frac{g_{\sigma}}{m_{\sigma}}\right)^{2}\left[-b M\left(g_{\sigma} \sigma\right)^{2}-c\left(g_{\sigma} \sigma\right)^{3}\right. \\
\left.+\sum_{B} \frac{\chi_{\sigma B}}{2 \pi^{2}} \int_{0}^{k_{F, B}} \frac{M_{B}^{*}(\sigma)}{\sqrt{k^{2}+M_{B}^{* 2}}} k^{2} d k\right],
\end{gathered}
$$

where we defined the ratio between meson-hyperon and meson-nucleon coupling constants as

$$
\chi_{(\sigma, \omega, \varrho), B} \equiv \frac{g_{(\sigma, \omega, \varrho), B}}{g_{(\sigma, \omega, \varrho)}} .
$$

In these equations, the baryon source terms have been replaced by their ground-state values.

The corresponding equations for baryon number and electric charge conservation are

$$
\rho=\sum_{B} \frac{k_{F, B}^{3}}{3 \pi^{2}}
$$

and

$$
\sum_{B} q_{e, B} \frac{k_{F, B}^{3}}{3 \pi^{2}}-\sum_{l} \frac{k_{F, l}^{3}}{3 \pi^{2}}=0 .
$$

The baryon chemical potentials, $\mu_{B}(k)$, correspond to eigenvalues of the Dirac equation (7):

$$
\mu_{B}(k)=g_{\omega B} \omega_{0}+g_{\varrho B} \varrho_{03} I_{3 B}+\sqrt{k_{F, B}^{2}+M_{B}^{*}(\sigma)^{2}} .
$$

In these expressions, $I_{3 B}$ is the isospin projection of baryon charge states $B$, and $k_{F, B}$ is the Fermi momentum of species $B$.

The EOS is obtained from the ground-state expectation value of the time and space components of the diagonal energy-momentum tensor. The energy density and pressure of the system are given, in the BB model, by

$$
\begin{aligned}
\varepsilon= & \frac{1}{3} b M\left(g_{\sigma} \sigma\right)^{3}+\frac{1}{4} c\left(g_{\sigma} \sigma\right)^{4}+\frac{1}{2} m_{\sigma}^{2} \sigma^{2}+\frac{1}{2} m_{\omega}^{2} \omega_{0}^{2} \\
& +\frac{1}{2} m_{\rho}^{2} \varrho_{03}^{2}+\sum_{B} \frac{1}{\pi^{2}} \int_{0}^{k_{F, B}} \sqrt{k^{2}+M_{B}^{* 2}} k^{2} d k \\
& +\sum_{l} \frac{1}{\pi^{2}} \int_{0}^{k_{F, l}} \sqrt{k^{2}+m_{l}^{2}} k^{2} d k
\end{aligned}
$$

$$
\begin{aligned}
p= & -\frac{1}{3} b M\left(g_{\sigma} \sigma\right)^{3}-\frac{1}{4} c\left(g_{\sigma} \sigma\right)^{4}-\frac{1}{2} m_{\sigma}^{2} \sigma^{2}+\frac{1}{2} m_{\omega}^{2} \omega_{0}^{2} \\
& +\frac{1}{2} m_{\rho}^{2} \varrho_{03}^{2}+\frac{1}{3} \sum_{B} \frac{1}{\pi^{2}} \int_{0}^{k_{F, B}} \frac{k^{4} d k}{\sqrt{k^{2}+M_{B}^{* 2}}} \\
& +\frac{1}{3} \sum_{l} \frac{1}{\pi^{2}} \int_{0}^{k_{F, l}} \frac{k^{4} d k}{\sqrt{k^{2}+m_{l}^{2}}}
\end{aligned}
$$

In the following we present the results obtained with this model.

\section{B. Results}

In numerical calculations with the BB model we have considered matter with and without hyperons, in order to understand how these strange species affect the neutron star properties.

\section{Matter with nucleons, hyperons, and leptons}

Figure 1, panels (a)-(d), shows baryon and lepton populations and field strengths as functions of the total baryon density for two different ratios: $\chi=\sqrt{2 / 3}^{1}$ and $\chi=1$ (universal coupling). From expression (14) for the baryon chemical potential we can see that the charge term in the eigenvalue determines whether a species is charge favored or unfavored, and the isospin term determines whether a species is isospin favored or not. Baryons with the same sign of the electric charge as the proton are unfavored; baryons with the same sign of its isospin projection are favored (note that $g_{\varrho} \varrho_{03}$ $<0)$.

At high densities $\left(\rho \sim 0.8 \mathrm{fm}^{-3}\right)$ the $\Lambda$ hyperon becomes the most populous species for the case $\chi=\sqrt{2 / 3}$ [Fig. 1(a)]. In Fig. 1(b) we see that the electron chemical potential reaches a maximum value at $\sim 200 \mathrm{MeV}$ and begins to decrease due to the reduction of the electron population. At $\rho$ $\sim 1.5 \mathrm{fm}^{-3}$ the nucleon effective mass is still at $200 \mathrm{MeV}$.

In Fig. 1(c), we see that increasing the meson-hyperon coupling constant (from $\sqrt{2 / 3}$ to 1 ) corresponds to an early emergence of the particles. In comparison with the results of Fig. 1(a), we can see that the leptons have a greater population in this case and that the neutron population remains always as the most important in the system. The electron chemical potential and $-g_{\varrho} \varrho_{03}$ saturates around $200 \mathrm{MeV}$ in Fig. 1(d), and the nucleon effective mass behaves similarly to the case $\chi=\sqrt{2 / 3}$.

\section{Matter with nucleons and leptons}

In the sequence of the analysis of the results of Fig. 1, panel (e) shows baryon and lepton populations and the corresponding chemical potentials when we exclude hyperon degrees of freedom. In this case, since charge neutrality is kept only by the $p^{+}, e^{-}$, and $\mu^{-}$particles, the lepton population increases in the domain of densities shown in the fig-

\footnotetext{
${ }^{1}$ This choice is based on a quark counting of the baryons [13].
} 
a)
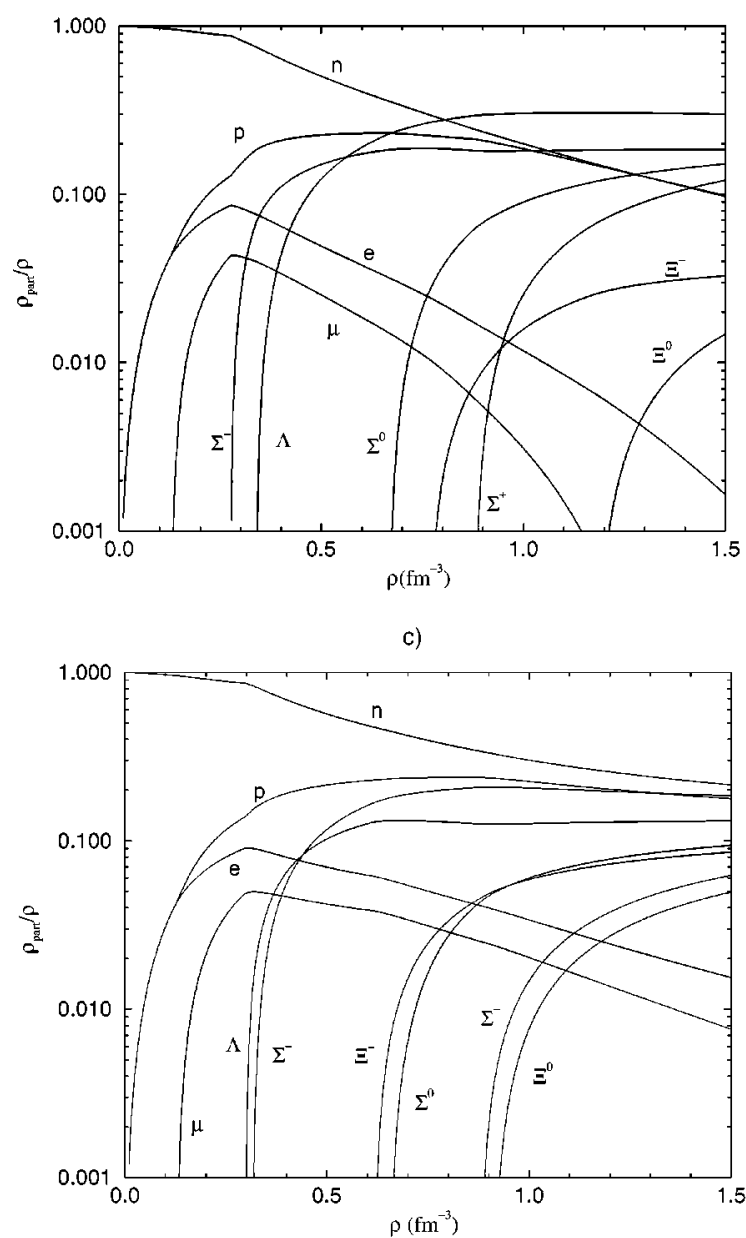

e)

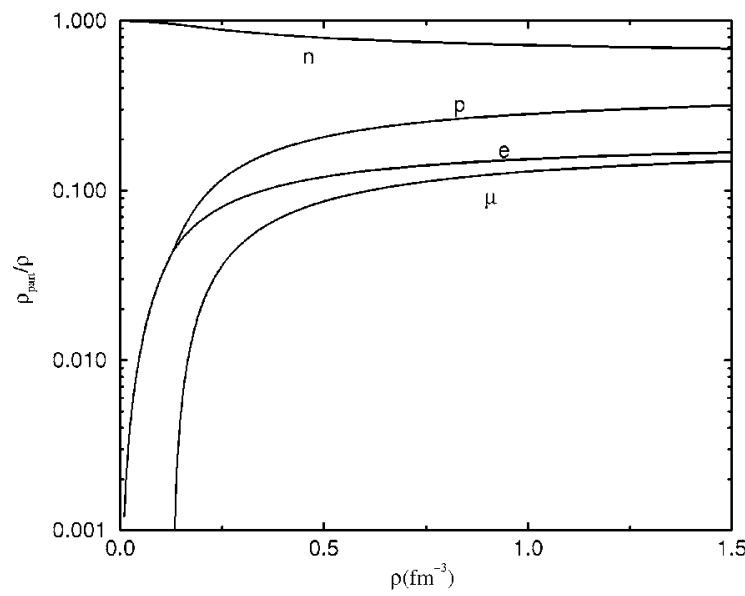

b)

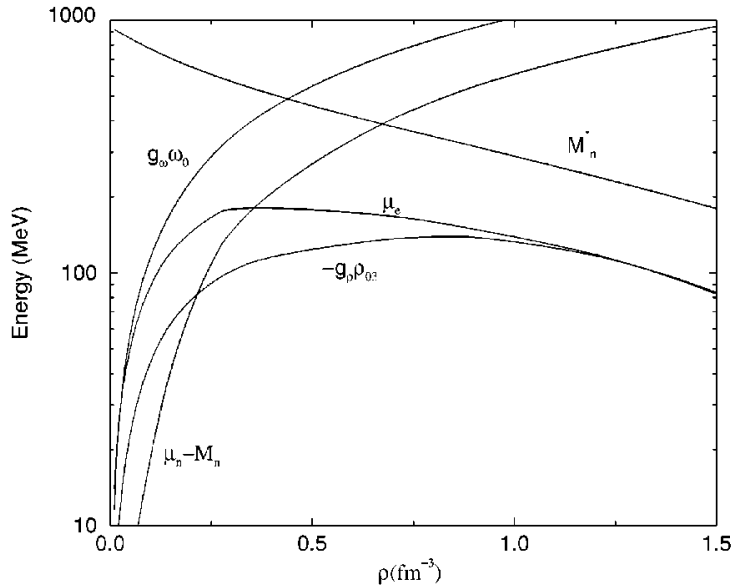

d)

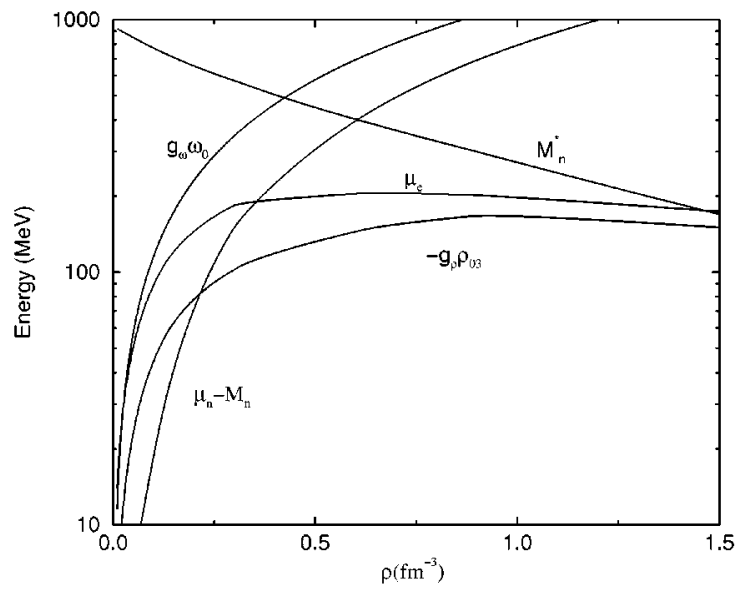

f)

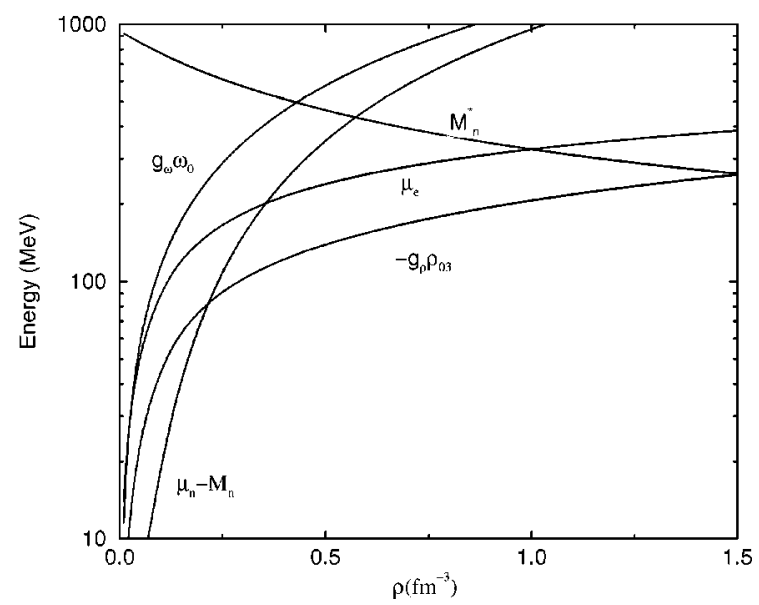

FIG. 1. Fermionic populations and field strengths in the BB model for matter with hyperons [panels (a) and (b) correspond to $\chi$ $=\sqrt{2 / 3}$ and panels (c) and (d) to $\chi=1]$ and without them [panels (e) and (f)].

ure. Also, we note the increase of the nucleon effective mass, which means a less intense scalar field when compared to the previous cases. This indicates that the introduction of hyperons enhances the scalar meson condensation.

\section{Neutron star properties}

We are able now to find numerical results for the EOS using Eqs. (15) and (16). However, this EOS corresponds to neutron star matter densities $\left(10^{13}-10^{15} \mathrm{~g} / \mathrm{cm}^{3}\right)$ and should 
a)

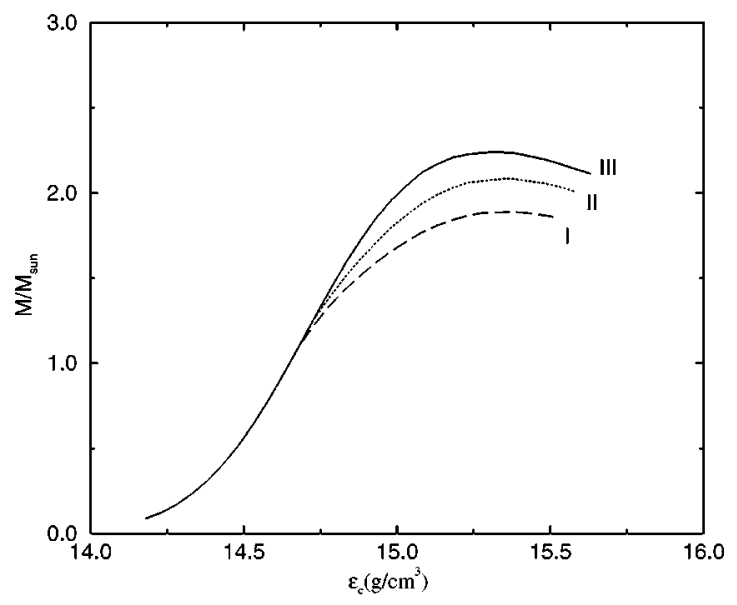

b)

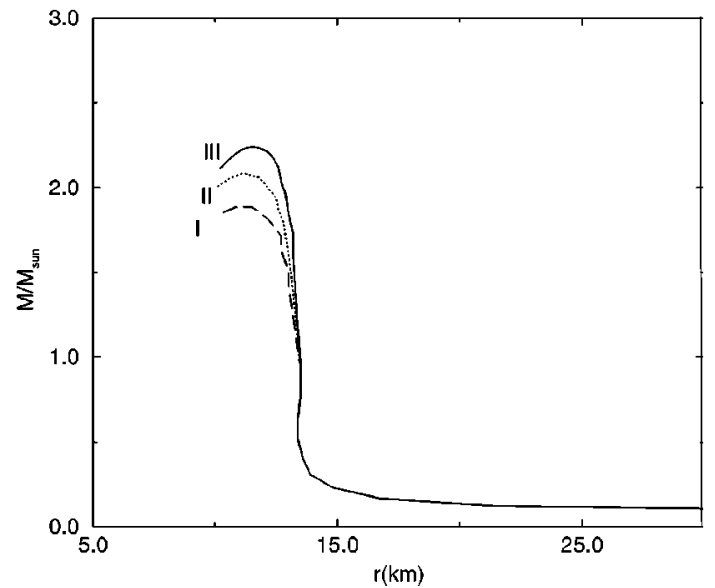

FIG. 2. Panel (a): Neutron star mass as a function of the central density for matter with (curve I corresponds to $\chi=\sqrt{2 / 3}$, and curve II to $\chi=1$ ) and without (curve III) hyperons. Panel (b): mass-radius relation [same labels as in panel (a)].

be supplemented by EOS's from other models for subnuclear densities: we adopt the approach developed in Ref. [14] in the density interval $2 \times 10^{3}-1 \times 10^{11} \mathrm{~g} / \mathrm{cm}^{3}$, and in the range $1 \times 10^{11}-2 \times 10^{13} \mathrm{~g} / \mathrm{cm}^{3}$ we use the EOS presented in Ref. [15]. Combining these EOS's with the TolmanOppenheimer-Volkoff (TOV) equations [16,17], we may determine the mass of a neutron star as a function of its central density; the radius $R$ is obtained with the condition that the pressure is null at the surface of the star, $p(R)=0$. We have found values for the mass and radius of different neutron star sequences as a function of the central density $\varepsilon_{c}$.

The conversion of nucleons into hyperons reduces the Fermi pressure associated with the baryons, softening the equation of state and lowering, as a consequence, the maximum mass of a neutron star sequence. In Fig. 2(a), we see the neutron star mass and central density relations for the situations analyzed above. The results indicate, as expected, that the presence of hyperons causes a diminution of the maximum mass of a neutron star sequence.

Typical results for the mass-radius relation are shown in Fig. 2(b). Our results indicate that neutron populations are, in general, dominant. However, in the case with $\chi=\sqrt{2 / 3}$ we obtained important contribution from the $\Lambda$ hyperons in the inner regions of the star. Moreover, we obtained $\sim 11 \mathrm{~km}$ for the radius of the star with hyperons, and $\sim 12 \mathrm{~km}$ without these particles.

\section{MODELS WITH DERIVATIVE COUPLINGS}

The BB model has two additional coupling constants $b$ and $c$, associated with self-interactions of the neutral meson scalar field. This allows a very good description of two important properties of nuclear matter, the compression modulus and the nucleon effective mass, which concern the highdensity behavior of the equation of state. However, some authors argued that the model suffers from a serious problem: the constant $c$ has negative values for several entries of $M^{\star}$ and $K$, allowing the energy density to become unbounded from below for large values of the scalar meson mean field, leading to unphysical behavior [18].

In the derivative coupling model, introduced in 1990 [7], the deficiencies of the original Walecka approach were eliminated at the cost of making the theory nonrenormalizable. ZM models were used in the description of static properties of neutron stars [19], $\Delta$ excitations in nuclear matter [20], bulk properties of finite nuclei [10], in-medium quark and gluon condensates and restoration of chiral symmetry [21], and thermodynamic properties of nuclear matter $[22,23]$.

The authors of Ref. [7] presented two additional versions of the derivative coupling model. These three models are known as ZM, ZM2, and ZM3 [8,9]. Concerning the description of static properties of nuclear matter, the ZM2 model does not exhibit fundamental differences from the ZM model, and will not be considered in the present study.

The Lagrangian density of the ZM and ZM3 models can be written in the general form

$$
\begin{aligned}
\mathcal{L}= & \bar{\psi}\left[i \gamma_{\mu}\left(\partial^{\mu}+i g_{\omega}^{\star} \omega^{\mu}\right)-\left(M-g_{\sigma}^{\star} \sigma\right)\right] \psi+\frac{1}{2}\left(\partial_{\mu} \sigma \partial^{\mu} \sigma\right. \\
& \left.-m_{\sigma}^{2} \sigma^{2}\right)-\frac{1}{4} \omega_{\mu \nu} \omega^{\mu \nu}+\frac{1}{2} m_{\omega}^{2} \omega_{\mu} \omega^{\mu}
\end{aligned}
$$

with $g_{\sigma}^{\star}=m^{\star} g_{\sigma}, g_{\omega}^{\star}=m^{\star} g_{\omega}$, where ${ }^{2}$

$$
m^{*} \equiv\left(1+\frac{g_{\sigma} \sigma}{M}\right)^{-1}
$$

Expression (17) reproduces the Lagrangian density of the Walecka, ZM, and ZM3 models with the following replacements [9]:

$$
\begin{array}{cc}
\text { Walecka: } \quad g_{\sigma}^{\star} \rightarrow g_{\sigma}, \quad g_{\omega}^{\star} \rightarrow g_{\omega}, \\
\text { ZM: } \quad g_{\sigma}^{\star} \rightarrow m^{*} g_{\sigma}, \quad g_{\omega}^{\star} \rightarrow g_{\omega}, \\
\text { ZM3: } \quad g_{\sigma}^{\star} \rightarrow m^{*} g_{\sigma}, \quad g_{\omega}^{\star} \rightarrow m^{*} g_{\omega} .
\end{array}
$$

\footnotetext{
${ }^{2}$ Note that in the ZM models we have $m^{\star} M \equiv M-g_{\sigma}^{\star} \sigma$.
} 
TABLE I. Values of the coupling constants, the nucleon effective mass, the compression modulus of the nuclear matter, and scalar and vector potentials at saturation density (ZM and ZM3 models).

\begin{tabular}{lccccccc}
\hline \hline Model & $\begin{array}{c}\left(g_{\sigma} / m_{\sigma}\right)^{2} \\
\left(\mathrm{fm}^{2}\right)\end{array}$ & $\begin{array}{c}\left(g_{\omega} / m_{\omega}\right)^{2} \\
\left(\mathrm{fm}^{2}\right)\end{array}$ & $\begin{array}{c}\left(g_{\rho} / m_{\rho}\right)^{2} \\
\left(\mathrm{fm}^{2}\right)\end{array}$ & $M^{\star} / M$ & $\begin{array}{c}K \\
(\mathrm{MeV})\end{array}$ & $\begin{array}{c}S \\
(\mathrm{MeV})\end{array}$ & $\begin{array}{c}V \\
(\mathrm{MeV})\end{array}$ \\
\hline ZM & 7.94 & 2.84 & 5.23 & 0.85 & 224 & -140 & 84 \\
ZM3 & 19.57 & 13.45 & 9.06 & 0.71 & 159 & -267 & 204 \\
\hline \hline
\end{tabular}

The resulting field equations are

$$
\begin{gathered}
{\left[i \gamma_{\mu} \partial^{\mu}-\left(M-g_{\sigma}^{\star} \sigma\right)-g_{\omega}^{\star} \gamma_{\mu} \omega^{\mu}\right] \psi=0,} \\
\partial_{\nu} \omega^{\nu \mu}+m_{\omega}^{2} \omega^{\mu}=g_{\omega}^{\star} \bar{\psi} \gamma^{\mu} \psi, \\
\left(\partial_{\mu} \partial^{\mu}+m_{\sigma}^{2}\right) \sigma=\frac{\partial g_{\sigma}^{\star} \sigma}{\partial \sigma} \bar{\psi} \psi-\frac{\partial g_{\omega}^{\star}}{\partial \sigma} \bar{\psi} \omega_{\mu} \gamma^{\nu} \psi .
\end{gathered}
$$

A peculiar aspect of the ZM3 model is the coupling between the scalar and vector meson fields [8], a kind of coupling which is not present in other QHD models (Walecka, BB, and $\mathrm{ZM}$ ). The scalar and vector mean-field potentials are defined to be $S \equiv-g_{\sigma}^{\star} \sigma$ and $V \equiv g_{\omega}^{\star} \omega_{0}$. In these models, the expressions for the energy density and pressure are equivalent to the corresponding expressions (15) and (16) of the BB model, with $b=c=0$ and the replacements shown in Eq. (19).

The values of the scalar $\left(g_{\sigma} / m_{\sigma}\right)^{2}$ and vector $\left(g_{\omega} / m_{\omega}\right)^{2}$ coupling constants which reproduce the saturation density $\rho_{0}=0.15 \mathrm{fm}^{-3}$ and the saturation energy $B=-16 \mathrm{MeV}$, in the cases of the ZM and ZM3 models, are shown in Table I; the table also contains the values of the nucleon effective mass, the compression modulus of nuclear matter, the scalar and vector mean fields $S$ and $V$ for both models, and values for the isovector coupling constant $\left(g_{\varrho} / m_{\varrho}\right)^{2}$ which reproduce the symmetry energy coefficient $a_{4}=32.5 \mathrm{MeV}$. The ZM and ZM3 models produce higher values for the nucleon effective mass since the coupling constants $g_{\sigma}^{\star}$ and $g_{\omega}^{\star} d e$ crease with increasing density. These models also predict a softer equation of state when compared to the corresponding results for the Walecka and $\mathrm{BB}$ models (lower compression modulus).

After the work of Zimanyi and Moszkowski, many authors started to explore extensively the freedom in the choice of the meson-baryon interaction. Based on these works, in this paper we introduce a new class of models which enables us to make direct comparisons among the properties of nuclear matter and neutron stars.

\section{A CLASS OF NONLINEAR RELATIVISTIC MODELS}

In this section we present a new class of relativistic hadronic models which exhibits a nonlinear parametrization of the intensity of the meson couplings, and incorporates some QHD models found in the literature. We study, through this comprehensive approach, the influence of nonlinear mesonnucleon couplings in the nucleon effective mass, the compression modulus of nuclear matter, and static properties of neutron stars.

\section{A. Phenomenological Lagrangians}

Koepf et al. [24] studied the contribution of the term $\mathcal{L}_{\sigma N}=M^{\star}(\sigma) \bar{\psi} \psi$ using several nonlinear functional forms for $M^{\star}(\sigma)$; Glendenning et al. [19] analyzed a coupling term of the type $M^{\star}=M-g_{\sigma} \sigma /\left(1+g_{\sigma} \sigma / 2 M\right)$. In these studies the authors assumed, at first order in $g_{\sigma} \sigma / M$, a similar expression for the nucleon effective mass as introduced by Walecka: $M^{*} / M \sim 1-g_{\sigma} \sigma / M$. These different models just add scalar self-coupling corrections terms to the corresponding expression of the Walecka model.

On the basis of the various approaches found in the literature, we propose a new phenomenological Lagrangian with a nonlinear parametrization, through mathematical constraints ( $\lambda, \beta$, and $\gamma$ parameters), of the analytical form of the meson-baryon couplings,

$$
\begin{aligned}
\mathcal{L}= & \sum_{B} \bar{\psi}_{B}\left[i \gamma_{\mu} \partial^{\mu}-\left(M_{B}-g_{\sigma B}^{\star} \sigma\right)-g_{\omega B}^{\star} \gamma_{\mu} \omega^{\mu}\right. \\
& \left.-\frac{1}{2} g_{\varrho B}^{\star} \gamma_{\mu} \tau \cdot \varrho^{\mu}\right] \psi_{B}+\sum_{\lambda} \bar{\psi}_{\lambda}\left[i \gamma_{\mu} \partial^{\mu}-m_{\lambda}\right] \psi_{\lambda} \\
& +\frac{1}{2}\left(\partial_{\mu} \sigma \partial^{\mu} \sigma-m_{\sigma}^{2} \sigma^{2}\right)-\frac{1}{4} \omega_{\mu \nu} \omega^{\mu \nu}+\frac{1}{2} m_{\omega}^{2} \omega_{\mu} \omega^{\mu} \\
& -\frac{1}{4} \varrho_{\mu \nu} \cdot \varrho^{\mu \nu}+\frac{1}{2} m_{\varrho}^{2} \varrho_{\mu} \cdot \varrho^{\mu}
\end{aligned}
$$

where

$$
g_{\sigma B}^{\star} \equiv m_{\lambda B}^{\star} g_{\sigma}, \quad g_{\omega B}^{\star} \equiv m_{\beta B}^{\star} g_{\omega}, \quad g_{\varrho B}^{\star}=m_{\gamma B}^{\star} g_{\varrho}
$$

and

$$
m_{n B}^{\star} \equiv\left(1+\frac{g_{\sigma} \sigma}{n M_{B}}\right)^{-n}, \quad n=\lambda, \beta, \gamma
$$

In these expressions, we assume $\lambda, \beta$, and $\gamma$ as real and positive numbers, since this is the range of best phenomenology. As discussed in the Sec. IV, essentially what was done is the introduction of a rescaling of the scalar and vector coupling terms of the Walecka model. For instance, in the case of the scalar contribution we have made the replacement

$$
g_{\sigma} \sigma \bar{\psi} \psi \rightarrow g_{\sigma}^{\star} \bar{\psi} \sigma \psi=\frac{g_{\sigma} \sigma}{\left(1+\frac{g_{\sigma} \sigma}{\lambda M}\right)^{\lambda}} \bar{\psi} \psi
$$


TABLE II. Values of $\lambda, \beta$, and $\gamma$ for different QHD models.

\begin{tabular}{llll}
\hline \hline Model & $\lambda$ & $\beta$ & $\gamma$ \\
\hline Walecka & 0 & 0 & 0 \\
ZM & 1 & 0 & 0 \\
ZM3 & 1 & 1 & 1 \\
\hline \hline
\end{tabular}

Similar interaction terms may be associated to the vector and isovector sector of the Lagrangian density. Note that we have assumed a universal coupling by setting $g_{(\sigma, \omega, \varrho) B}$ $\rightarrow g_{(\sigma, \omega, \varrho)}$.

Table II exhibits the correspondence between this model and the other models discussed in this work with specific values of $\lambda, \beta$, and $\gamma$. One of the main intentions of the present study is to consider values of these parameters which give better results for nuclear matter and neutron star properties when compared to the corresponding results of the traditional models discussed in this work. As far as we know, the first extensions of the ZM-like models to applications to neutron star matter with the inclusion of hyperons $\left(\Lambda, \Sigma^{-}\right)$ and leptons was done in Ref. [25]. Here we consider these known models as well as intermediate values of the parameters of our nonlinear coupling, to obtain results for neutron star properties and relate them to nuclear matter saturation observables.

Using the mean-field approximation, the field equations in our approach become

$$
\begin{gathered}
{\left[i \gamma_{\mu} \partial^{\mu}-g_{\omega B}^{\star} \gamma_{0} \omega^{0}-\left(M_{B}-g_{\sigma B}^{\star} \sigma\right)\right] \psi_{B}=0} \\
g_{\omega} \omega_{0}=\left(\frac{g_{\omega}}{m_{\omega}}\right)^{2} \sum_{B} m_{\beta B}^{*} \rho_{B}, \\
g_{\varrho} \varrho_{03}=\left(\frac{g_{\varrho}}{m_{\varrho}}\right)^{2} \sum_{B} m_{\gamma B}^{*} I_{3 B} \rho_{B}, \\
-g_{\sigma} \sigma=\left(\frac{g_{\sigma}}{m_{\sigma}}\right)^{2}\left[\sum_{B}\left(\frac{F_{B}(\sigma)}{g_{\sigma}}\right) \varrho_{s, B}+g_{\omega} \omega_{0} \sum_{B}\left(\frac{G_{B}(\sigma)}{g_{\sigma} g_{\omega}}\right) \rho_{B}\right. \\
\left.+g_{\varrho} \varrho_{03} \sum_{B}\left(\frac{H_{B}(\sigma)}{g_{\sigma} g_{\varrho}}\right) I_{3 B} \rho_{B}\right],
\end{gathered}
$$

where $F_{B}(\sigma), G_{B}(\sigma)$, and $H_{B}(\sigma)$ are given by

$$
\begin{gathered}
\frac{F_{B}(\sigma)}{g_{\sigma}}=-m_{\lambda B}^{*}+\frac{g_{\sigma} \sigma}{M_{B}}\left(m_{\lambda B}^{*}\right)^{(\lambda+1) / \lambda}, \\
\frac{G_{B}(\sigma)}{g_{\sigma} g_{\omega}}=-\frac{\left(m_{\beta B}^{*}\right)^{(\beta+1) / \beta}}{M_{B}},
\end{gathered}
$$

and

$$
\frac{H_{B}(\sigma)}{g_{\sigma} g_{\varrho}}=-\frac{\left(m_{\gamma B}^{*}\right)^{(\gamma+1) / \gamma}}{M_{B}} .
$$

From the eigenvalues of the Dirac equation, the Fermi energy is

$$
\mu_{B}(k)=g_{\omega B}^{\star} \omega_{0}+g_{\varrho B}^{\star} \varrho_{03} I_{3 B}+\sqrt{k_{F, B}^{2}+\left(M_{B}-g_{\sigma B}^{\star} \sigma\right)^{2}} .
$$

The expressions for the scalar and vector potentials $(S)$ and $(V)$ are

$$
S=-m_{\lambda}^{*} g_{\sigma} \sigma, \quad V=m_{\beta}^{*} g_{\omega} \omega_{0} .
$$

We can see that this model allows some control on the intensity of the scalar and vector mesons mean-field potentials. For instance, to the variations of $\lambda$ between 0 and 1, keeping $\beta=\gamma=0$, we obtain values of $S, V, M^{*}$, and $K$ which correspond to the intermediate region of values of Walecka and ZM models. Similarly, for values of $\lambda, \beta$, and $\gamma$ between 0 and 1 , we can find intermediate results between the Walecka and the ZM3 models.

Indeed, the range of possible values for the parameters of the theory is not very large. Due to the form of the general coupling terms [see Eq. (25)], there occurs a rapid convergence to an exponential form. Taking $\lambda, \beta$, and $\gamma \rightarrow \infty$, we have

$$
\begin{gathered}
g_{\sigma B}^{\star} \longrightarrow e^{-g_{\sigma} \sigma / M_{B}} g_{\sigma}, \quad g_{\omega B}^{\star} \longrightarrow e^{-g_{\sigma} \sigma / M_{B} g_{\omega}}, \\
g_{\varrho B}^{\star} \longrightarrow e^{-g_{\sigma} \sigma / M_{B}} g_{\varrho} .
\end{gathered}
$$

As we will see below, for $\lambda$ and/or $\beta=\gamma>2$ the results of this model do not strongly differ from the results of the model with exponential coupling. In this work, we shall consider two cases.

(i) Scalar (case $S$ ): in this case we consider variations of $\lambda$ with $\beta=\gamma=0$; this case contains the results of the Walecka and ZM models.

(ii) Scalar-vector (case $S-V$ ): in this case we consider variations of $\lambda$, with $\beta=\gamma=\lambda$; Walecka and ZM3 models belong to this category.

Note that the models we are discussing may be uniquely specified by the $\lambda$ parameter. The Walecka model belongs to both categories, because in this model the mathematical parameters $\lambda, \beta$, and $\gamma$ are null; however, it does not present scalar-vector interaction contributions.

\section{B. Nuclear properties}

We determine the coupling constants $g_{\sigma, \omega, \varrho} / m_{\sigma, \omega, \varrho}$ in this model by following the same procedure presented in Sec. III. For each case, $S$ and $S-V$, we obtain numerical values for $g_{\sigma} / m_{\sigma}, g_{\omega} / m_{\omega}, M^{\star}$, and $K$ as functions of $\lambda$. Thus we can also determine $g_{\varrho} / m_{\varrho}$.

Figure 3 exhibits the dependence of the coupling constants on the $\lambda$ parameter; it is interesting to note the regular relative behavior of the coupling constants $\left(g_{\sigma} / m_{\sigma}\right)^{2}$ and $\left(g_{\omega} / m_{\omega}\right)^{2}$. The results indicate that the scalar case suffers a $\lambda$-dependent saturation process in a small range of values for this parameter. On the other hand, the results also reveal that the scalar-vector case exhibits a wider range of values of $\lambda$.

Figure 4 presents the relation between the compression modulus and the nucleon effective mass, $K \times M^{*} / M$, for the $S$ and $S-V$ cases. From the results, one can see that lower values of the compression modulus correspond to higher val- 




FIG. 3. Dependence of the coupling constants with the $\lambda$ parameter for the scalar (full lines) and scalar-vector (dotted lines) models.

ues of the nucleon effective mass: to understand this behavior one should remember that the repulsive mean-field vector potential $V$ is proportional to $M-M^{*}$. From the results found in the literature [26], the nucleon effective mass and the compression modulus should be in the range 0.6 $<M^{*} / M<0.7$ and $200<K<300 \mathrm{MeV}$. As stressed above, the scalar case leads to a narrow interval of values of $\lambda$ while the scalar-vector case has a broader range. Accordingly, case $S$ gives reasonable results in the range $\lambda \sim 0.05-0.07$ and case $S-V$ gives better results for $\lambda \sim 0.16-0.4$. The analysis also reveals the strong similarity between the results of ZMlike models $(\lambda=1)$ and those with exponential couplings $(\lambda \rightarrow \infty)$.

\section{Neutron star properties}

In this section we consider the determination of neutron stars properties using our class of nonlinear models with the inclusion of hyperon and lepton degrees of freedom. Following the same procedure of Sec. III, we solve a system of transcendental equations taking into account chemical equilibrium, baryon number, and electric charge conservation

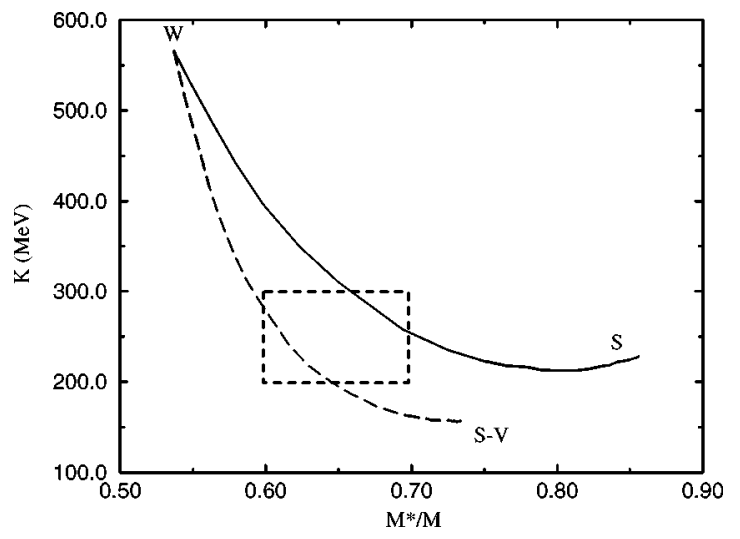

FIG. 4. Comparison of the compression modulus of nuclear matter $K$ with the ratio $M * / M$ for the $S$ and $S-V$ cases. The box shows the range of accepted values.

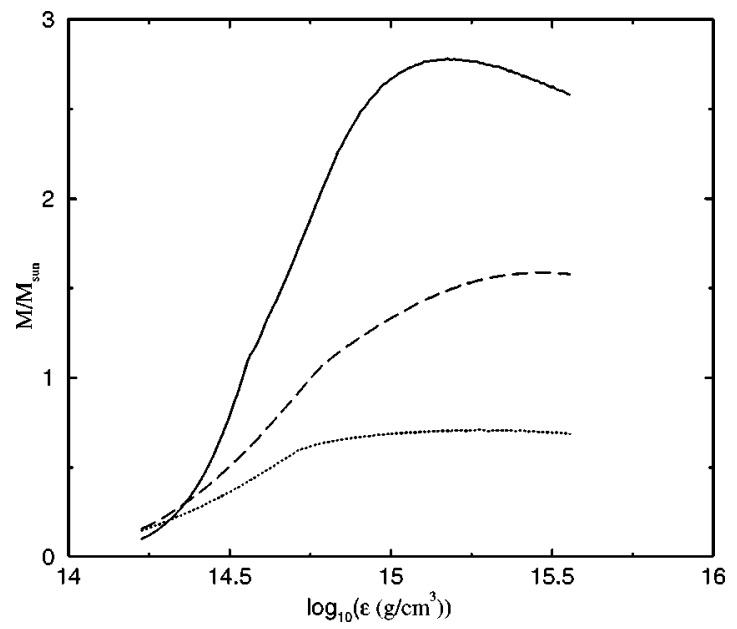

FIG. 5. Neutron star mass as a function of the central density in Walecka (solid line), ZM (dashed line), and ZM3 (dotted line) models.

and the equation for the $\sigma$ field. We then obtain the EOS for our system. The resulting expressions for the energy density and pressure are, again, similar to Eqs. (15) and (16) but with $b=c=0$ and $M_{B}^{\star}=M_{B}-g_{\sigma B}^{\star} \sigma$. Combining this EOS with the TOV equations, we obtain values for static properties of neutron stars (mass, radius, and baryon composition, among others) as functions of the central density.

Here we explicitly present the neutron star phenomenology for the Walecka and Zimanyi-Moszkowski models, namely, the original ZM and also the variant ZM3 model. The predictions for neutron star masses as a function of the central density in Walecka, ZM, and ZM3 models are shown in Fig. 5. The ZM model predicts a maximum mass of approximately $1.6 M_{\odot}$, in the limit of acceptability for the mass of a pulsar. In particular, the ZM3 model is very soft, and predicts a very small maximum neutron star mass, $\sim 0.72 M_{\odot}$.

It may be surprising, at first glance, that the maximum neutron star mass for the Walecka model with hyperons $\left(2.77 M_{\odot}\right)$ exceeds the well-known result $\left(2.6 M_{\odot}\right)$ found in Ref. [4] for stars composed of just neutrons, since the addition of other particles softens the EOS, lowering the resulting star mass. This apparent contradiction can be explained by the extreme sensibility of this kind of theory on the specific choice of the values of the binding energy and saturation density. The authors cited above used $B=-15.75 \mathrm{MeV}$ and $\rho_{0}=0.19 \mathrm{fm}^{-3}$; with this choice we were able to reproduce their results. However, with our choice for these quantities, which is widely used in the recent literature, for the mass of a star composed only by neutrons we obtain the value $3.05 M_{\odot}$, that is, a difference of almost a half solar mass. Using the constants from [4] $\left(a_{4}=33.6 \mathrm{MeV}\right)$, we obtain $2.33 M_{\odot}$ for the mass of a neutron star with the inclusion of hyperons and leptons. In this way, extrapolation for neutron star densities from the fitting of $B$ and $\rho_{0}$ at saturation needs more precision in the choice of the values for these quantities.

Figure 6 shows the behavior of the chemical potentials and field intensities only for Walecka and ZM models, since 
a)

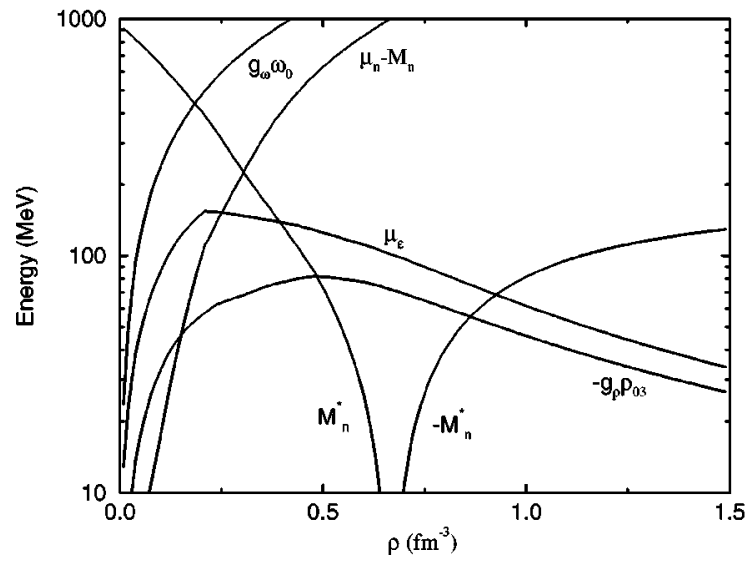

b)

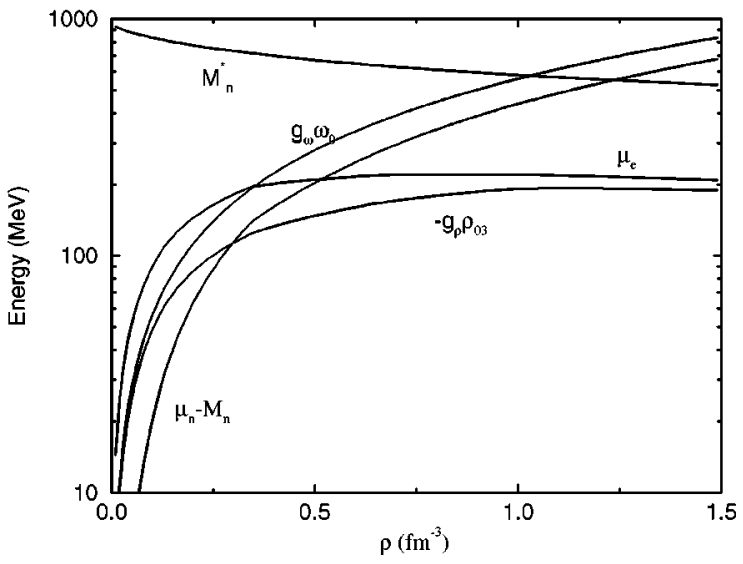

FIG. 6. Chemical potentials and field intensities in the Walecka [panel (a)] and ZM [panel (b)] models.

the ZM3 model gives a poor description of neutron star masses. These results should be compared to the corresponding one obtained with the BB model. We observe the same saturation of the electron chemical potential at $\sim 200 \mathrm{MeV}$ for the ZM and BB models with universal coupling [see Fig. $1(\mathrm{~d})]$. The known problem of negative effective mass [2729] manifests itself dramatically in panel (a) for the Walecka model. We discuss this point in Sec. V D.

In Fig. 7 the populations in a system consisting of hyperons, nucleons, and leptons, for the Walecka and ZM models, are shown. Walecka's baryonic distribution stabilizes after $\rho \sim 1.0 \mathrm{fm}^{-3}$, and all species appear up till $\rho \sim 0.7 \mathrm{fm}^{-3}$, which is approximately the density where $|S|$ exceeds $M$. The lepton populations never vanish in the ZM distribution, and even at $\rho \sim 1.2 \mathrm{fm}^{-3}$ baryonic species are emerging. Essentially, these differences are due to the strength of the scalar potential in these two models. Since a particle is created only when

$$
q_{B} \mu_{n}-q_{e, B} \mu_{e} \geqslant g_{\omega B}^{\star} \omega_{0}+g_{\rho B}^{\star} \varrho_{03} I_{3 B}+\left(M_{B}-g_{\sigma B}^{\star} \sigma\right),
$$

a large scalar field favors the early emergence of particles. In

a)

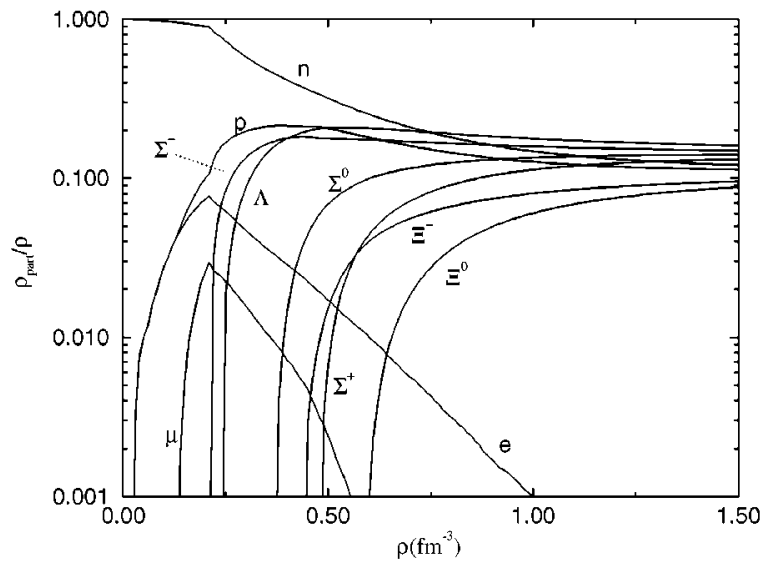

Fig. 8 the predictions of the ZM model for the radial distribution of the different lepton and baryon species is presented.

We now analyze our new class of models allowing variations of the $\lambda$ parameter. Tables III and IV show results for the radius, redshift $(z)$, and hyperon/baryon ratio for the maximum mass of a neutron star sequence for a given $\lambda$; we present some nuclear matter properties as well. In Fig. 9 we show the dependence of the maximum mass with this parameter. One can see that some models corresponding to the $S-V$ case predict very small neutron star masses, lower than the masses of all pulsars found until now. We again observe the similarity of predictions associated with the ZM model compared to the one with exponential couplings.

Figure 10 exhibits the dependence of the maximum mass of a neutron star of a sequence with the compression modulus and nucleon effective mass at saturation density in the $S$ and $S$ - $V$ cases. In both cases, in general, a less compressible matter (higher $K$ ) corresponds to a higher maximum neutron star mass. Weaker scalar potentials correspond to higher values of the nucleon effective mass; since the former is also directly related to the compression modulus, the maximum

b)

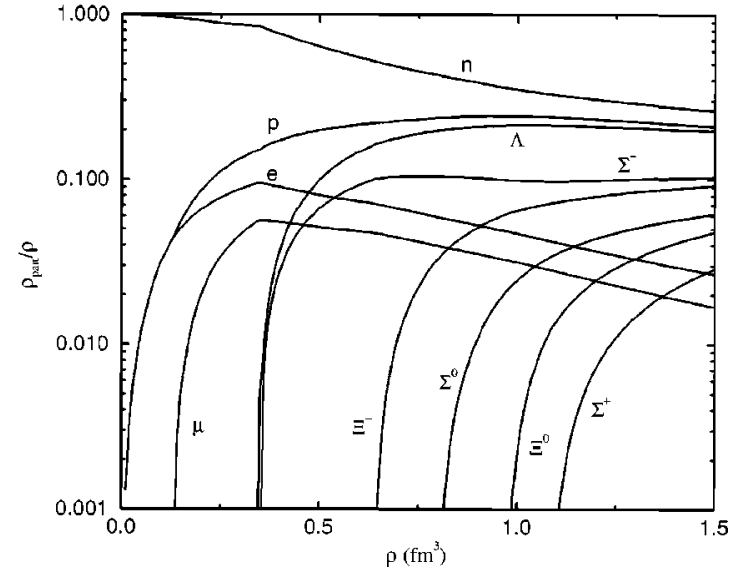

FIG. 7. Baryon and lepton populations for the Walecka [panel (a)] and ZM [panel (b)] models. 


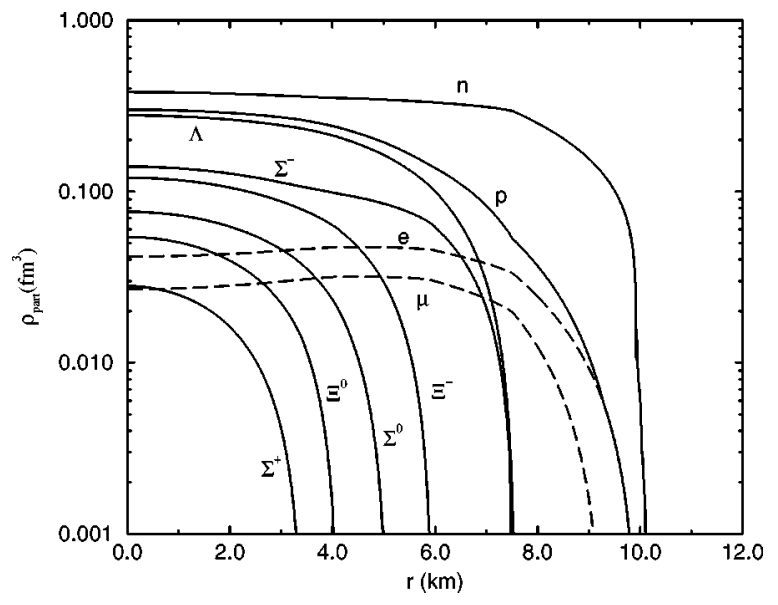

FIG. 8. Radial distribution of the different leptonic and baryonic species in the ZM model.

neutron star mass decreases with $M^{\star}$. In spite of the fact that the two cases represent different descriptions of the neutron star problem, the figure shows that, for a fixed value of the compression modulus there correspond very close values of the neutron star mass; opposite to this, for different values of the star mass there corresponds the same value of the nucleon effective mass. This result indicates that the predictions of neutron star masses based mainly on the compression modulus are more model independent than those based on the nucleon effective mass. From Table IV, we see that, for $\lambda<0.5$ in the $S-V$ case, we have obtained negative values for the nucleon effective mass, which corresponds to a density region for which the strength of the scalar condensate exceeds the free mass of the nucleon, $|S|>M$.

\section{Negative effective mass}

The nucleon effective mass is a dynamical quantity and expresses the screening of the baryon masses by the scalar meson condensate. In our approach, by analyzing the general expression for the baryon effective mass,

$$
M_{B}^{\star}=M_{B}-\frac{g_{\sigma} \sigma}{\left[1+g_{\sigma} \sigma /(\lambda M)\right]^{\lambda}},
$$

in the limit $g_{\sigma} \sigma \rightarrow \infty$, we see that only in case $\lambda \geqslant 1$ this quantity does not vanish or become negative. Some internal constraints in the theory can avoid this, as in the well-known case of the Walecka model without hyperons [4]. However, as we add more and more baryonic species we open the possibility for the scalar potential $|S|$ to become greater than the free masses.

Let us introduce the scalar field equation in Eq. (37), for the Walecka case, to obtain a better understanding of the problem; we obtain

$$
M_{B}^{\star}=M_{B}-\sum_{B^{\prime}} \frac{g_{\sigma}^{2}}{m_{\sigma}^{2}} \frac{M_{B^{\prime}}^{\star}}{\pi^{2}} \int \frac{k^{2} d k}{\sqrt{k^{2}+M_{B^{\prime}}^{\star}}}
$$

or

TABLE III. Stellar properties for the $S$ case: $\varepsilon_{c}$ is the central density, $M_{\star}$ is the star mass, $R_{\star}$ is the star radius, $S$ is the scalar potential in the star center, $z$ is a redshift; $Y / A$ is the hyperon/baryon ratio, and $N_{B T}$ is the total baryonic number. All these quantities are evaluated for the neutron star with the maximum mass in

\begin{tabular}{|c|c|c|c|c|c|c|c|c|c|}
\hline$\lambda$ & $\begin{array}{l}\ln \left(\varepsilon_{c}\right) \\
\mathrm{g} / \mathrm{cm}^{3}\end{array}$ & $\begin{array}{c}M_{\star} \\
\left(M_{\odot}\right)\end{array}$ & $\begin{array}{c}R_{\star} \\
(\mathrm{km})\end{array}$ & $\begin{array}{c}S \\
(\mathrm{MeV})\end{array}$ & $z$ & $Y / A$ & $\begin{array}{c}N_{B T} \\
\left(\times 10^{58}\right)\end{array}$ & $\begin{array}{c}K \\
(\mathrm{MeV})\end{array}$ & $M^{\star} / M$ \\
\hline 0 & 15.18 & 2.77 & 13.17 & 936 & 0.623 & 0.27 & 0.40 & 566 & 0.537 \\
\hline 0.03 & 15.24 & 2.56 & 12.39 & 934 & 0.597 & 0.30 & 0.36 & 396 & 0.598 \\
\hline 0.05 & 15.31 & 2.35 & 11.63 & 929 & 0.574 & 0.32 & 0.33 & 310 & 0.650 \\
\hline 0.07 & 15.38 & 2.17 & 10.89 & 923 & 0.554 & 0.34 & 0.30 & 258 & 0.694 \\
\hline 0.09 & 15.43 & 2.02 & 10.38 & 910 & 0.533 & 0.35 & 0.28 & 235 & 0.725 \\
\hline 0.11 & 15.47 & 1.91 & 9.98 & 896 & 0.515 & 0.36 & 0.26 & 223 & 0.749 \\
\hline 0.13 & 15.49 & 1.83 & 9.75 & 877 & 0.495 & 0.35 & 0.25 & 217 & 0.766 \\
\hline 0.15 & 15.52 & 1.77 & 9.59 & 857 & 0.479 & 0.35 & 0.24 & 216 & 0.779 \\
\hline 0.17 & 15.54 & 1.72 & 9.45 & 838 & 0.467 & 0.35 & 0.23 & 213 & 0.789 \\
\hline 0.20 & 15.53 & 1.68 & 9.48 & 807 & 0.446 & 0.33 & 0.23 & 212 & 0.798 \\
\hline 0.25 & 15.53 & 1.61 & 9.49 & 732 & 0.416 & 0.30 & 0.22 & 212 & 0.814 \\
\hline 0.30 & 15.52 & 1.59 & 9.61 & 669 & 0.399 & 0.27 & 0.21 & 214 & 0.822 \\
\hline 0.35 & 15.51 & 1.58 & 9.69 & 618 & 0.389 & 0.26 & 0.21 & 216 & 0.828 \\
\hline 0.40 & 15.51 & 1.58 & 9.73 & 580 & 0.385 & 0.25 & 0.21 & 218 & 0.833 \\
\hline 0.60 & 15.49 & 1.58 & 9.86 & 480 & 0.377 & 0.22 & 0.21 & 223 & 0.843 \\
\hline 1.00 & 15.47 & 1.59 & 9.98 & 401 & 0.372 & 0.20 & 0.21 & 224 & 0.850 \\
\hline 1.50 & 15.47 & 1.59 & 9.98 & 366 & 0.373 & 0.20 & 0.21 & 226 & 0.854 \\
\hline$\infty$ & 15.47 & 1.59 & 10.00 & 350 & 0.373 & 0.20 & 0.21 & 228 & 0.856 \\
\hline
\end{tabular}
the sequence. In addition, we have the compression modulus $K$ and the nucleon effective mass $M^{\star} / M$. 
TABLE IV. Stellar properties for the $S$ - $V$ case. Same correspondences as in Table III.

\begin{tabular}{cccccccccc}
\hline \hline$\lambda$ & $\begin{array}{c}\ln \left(\varepsilon_{c}\right) \\
\mathrm{g} / \mathrm{cm}^{3}\end{array}$ & $\begin{array}{c}M_{\star} \\
\left(M_{\odot}\right)\end{array}$ & $\begin{array}{c}R_{\star} \\
(\mathrm{km})\end{array}$ & $\begin{array}{c}S \\
(\mathrm{MeV})\end{array}$ & & $Y / A$ & $\begin{array}{c}N_{B T} \\
\left(\times 10^{58}\right)\end{array}$ & $\begin{array}{c}K \\
(\mathrm{MeV})\end{array}$ & $M^{\star} / M$ \\
\hline 0 & 15.18 & 2.77 & 13.17 & 936 & 0.623 & 0.27 & 0.40 & 566 & 0.537 \\
0.03 & 15.20 & 2.70 & 12.93 & 944 & 0.615 & 0.28 & 0.39 & 510 & 0.545 \\
0.05 & 15.22 & 2.63 & 12.64 & 954 & 0.610 & 0.29 & 0.38 & 458 & 0.554 \\
0.07 & 15.24 & 2.56 & 12.39 & 960 & 0.602 & 0.30 & 0.37 & 417 & 0.561 \\
0.09 & 15.27 & 2.50 & 12.12 & 969 & 0.598 & 0.31 & 0.36 & 387 & 0.567 \\
0.11 & 15.28 & 2.43 & 11.89 & 973 & 0.588 & 0.32 & 0.34 & 358 & 0.574 \\
0.13 & 15.30 & 2.37 & 11.68 & 977 & 0.579 & 0.33 & 0.33 & 339 & 0.579 \\
0.15 & 15.33 & 2.30 & 11.38 & 985 & 0.574 & 0.34 & 0.32 & 311 & 0.587 \\
0.17 & 15.35 & 2.24 & 11.16 & 986 & 0.563 & 0.34 & 0.31 & 293 & 0.594 \\
0.20 & 15.38 & 2.17 & 10.88 & 993 & 0.559 & 0.35 & 0.30 & 276 & 0.600 \\
0.30 & 15.51 & 1.83 & 9.58 & 1011 & 0.516 & 0.39 & 0.25 & 218 & 0.630 \\
0.35 & 15.56 & 1.70 & 9.09 & 1012 & 0.491 & 0.41 & 0.23 & 205 & 0.640 \\
0.40 & 15.62 & 1.57 & 8.60 & 1014 & 0.470 & 0.42 & 0.21 & 195 & 0.649 \\
0.60 & 15.62 & 1.07 & 8.08 & 891 & 0.282 & 0.35 & 0.14 & 169 & 0.682 \\
1.00 & 15.31 & 0.72 & 9.76 & 577 & 0.128 & 0.10 & 0.09 & 159 & 0.710 \\
1.50 & 15.18 & 0.67 & 10.21 & 468 & 0.113 & 0.04 & 0.08 & 156 & 0.728 \\
$\infty$ & 15.14 & 0.66 & 10.31 & 431 & 0.110 & 0.03 & 0.08 & 155 & 0.738 \\
\hline \hline
\end{tabular}

As we add more and more baryonic species, the negative term in the numerator of Eq. (38) becomes more important, and we open the possibility for the scalar potential $|S|$ to become greater than the free baryon masses. In fact, even if we had just considered nucleons, taking into account the difference in the neutron and proton masses, the negative term in the numerator would appear. However, since this difference is very tiny, a negative effective mass will emerge in practice only with the addition of hyperon degrees of freedom [29].

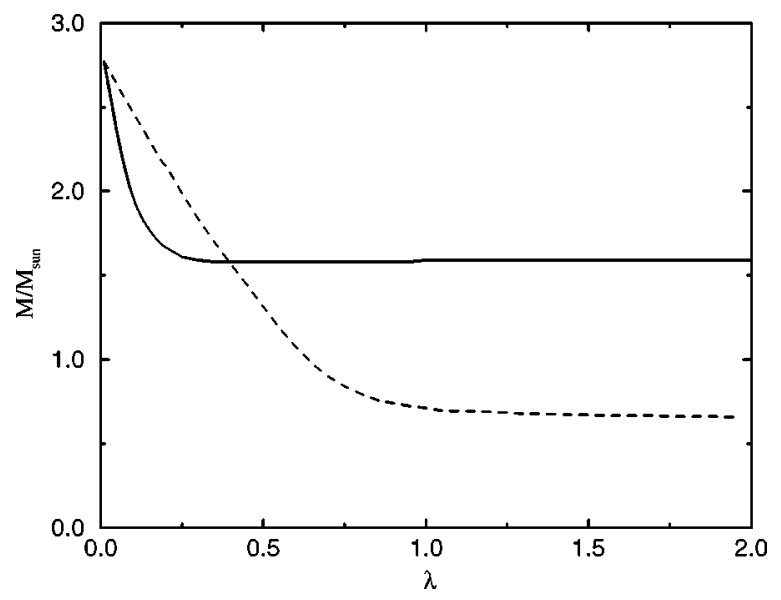

FIG. 9. Maximum mass of a neutron star sequence (universal coupling) as a function of the $\lambda$ parameter, for the $S$ (full line) and $S-V$ (long dashed line) cases.
We could interpret the vanishing of the effective mass as a signal of a transition to a quark-gluon plasma phase. However, we should remember that our Lagrangian model does not encompass these underlying degrees of freedom. Additionally, as pointed out in Ref. [30], at such high densities and strong meson fields we have already reached a critical density where the production of baryon-antibaryon pairs is favored. In fact, this behavior of the effective mass may in-

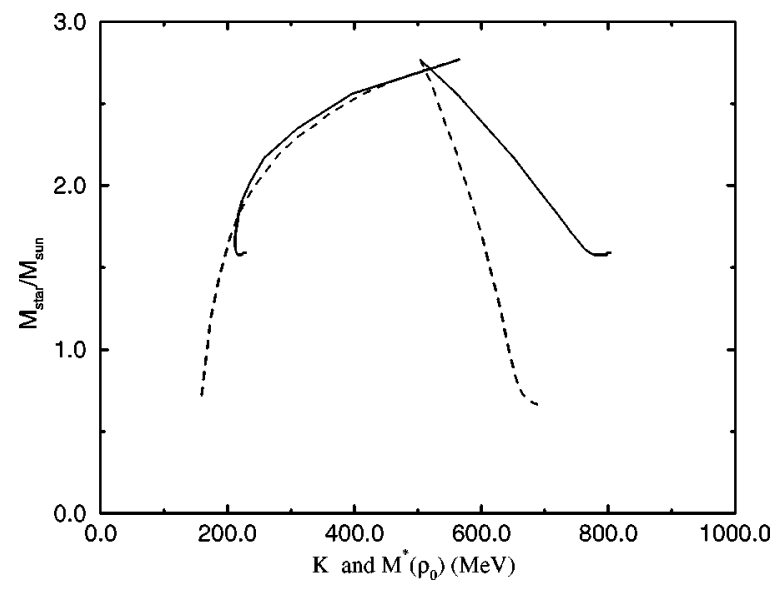

FIG. 10. Dependence of the maximum neutron star mass of a sequence with the compression modulus (left) and nucleon effective mass at saturation (right). The solid line corresponds to the $S$ case, and the dashed line to the $S-V$ case. 
dicate that the mean-field approximation is reaching the limits of its applicability.

Concerning the problem of the negative effective mass, we want to clarify a point which may appear naive but has led to misunderstandings. The integrals appearing in the QHD formalism always present a term $M^{\star 2}$ and, in this way, are symmetric with respect to positive or negative values of the effective mass. For example, let us take the integral related to the scalar density,

$$
I(t, m)=\int_{0}^{t} \frac{k^{2} d k}{\sqrt{k^{2}+m^{2}}} ;
$$

rigorously, the expression for $I(t, m)$ would involve the modulus of $m$ since $\sqrt{m^{2}}=|m|$ :

$$
\int_{0}^{t} \frac{k^{2}}{\sqrt{k^{2}+m^{2}}}=\frac{1}{2} t \sqrt{t^{2}+m^{2}}-\frac{1}{2} m^{2} \ln \left(\frac{t+\sqrt{t^{2}+m^{2}}}{|m|}\right) .
$$

Some authors assumed ad hoc the modulus of $M^{\star}$ in the above logarithm, when the problem of negative values appears. However, we can see from expression (40) that this emerges naturally from the symmetry of the integrals. Of course, different results would arise if we use $\left|M^{\star}\right|$ instead of $M^{\star}$ in other expressions, e.g., the cubic term in the energy density expression for the $\mathrm{BB}$ model, since this can be rewritten

$$
\frac{1}{3} b M\left(M-M^{\star}\right)^{3}
$$

Finally, we want to stress that, in spite of the interesting issue of the physical interpretation of a negative effective mass, mathematically the QHD models continue to work even with $M^{\star}<0$.

\section{SUMMARY AND CONCLUSIONS}

We have performed an analysis of the influence of nuclear matter properties on the structure of neutron stars, using a new class of QHD models with parametrized couplings among mesons and baryons. These couplings allow one to reproduce, through a suitable choice of mathematical parameters $(\lambda, \beta, \gamma)$, results of the Walecka and derivative coupling models such as ZM and ZM3 models. As we have shown above, this new class of relativistic models permits some control of the intensity of the scalar, vector, and, isovector meson mean-field potentials. In particular, we have scanned values of nuclear matter properties which correspond to intermediate region of predictions of the Walecka, ZM, and ZM3 models.

In this new class of models we considered two cases, the scalar case (which contains the results of the Walecka and ZM models) and the scalar-vector case (the ZM3 model belongs to this category). The exponential coupling models are obtained when we take the limit of the mathematical parameters going to infinity.

For nuclear matter, the $S$ case gives reasonable results in the range $\lambda \sim 0.05-0.07$ and the $S-V$ case gives better results for $\lambda \sim 0.16-0.4$. We showed results, allowing a variation of $\lambda$, for the radius, redshift $(z)$ and hyperon/baryon ratio for the maximum mass of a neutron star sequence, considering hyperon, nucleon, and lepton degrees of freedom. Some models corresponding to the $S$ - $V$ case predict very small neutron star masses. The ZM model gives a maximum mass of approximately $1.6 M_{\odot}$ for a neutron star, while the ZM3 model, being too soft, leads to $\sim 0.72 M_{\odot}$ as the limiting mass. We have also pointed out the strong similarity between the results of ZM-like models and those with exponential couplings.

The sensibility of this approach to the specific choice of $B$ and $\rho_{0}$ was noted, with differences of the order of a half solar mass. Thus and extrapolation for neutron star densities from the fitting of $B$ and $\rho_{0}$ at saturation needs more precision in the choice of the values for these quantities.

For some values of $\lambda$ in the $S-V$ case, we have obtained negative values for the nucleon effective mass. We have shown how the differences in the baryonic bare masses explain this result; as discussed in the text, in practice the inclusion of hyperons is responsible for this behavior. These results indicate the existence of very intense scalar condensates in the interior region of neutron stars.

\section{ACKNOWLEDGMENTS}

This work was supported by CNPq.

\section{APPENDIX: CHEMICAL EQUILIBRIUM}

Chemical reactions, e.g., $A+B \rightleftharpoons C+D$, can be expressed in general, as a symbolic linear combination of its components [31]:

$$
\sum_{i} \nu_{i} A_{i}=0
$$

For example, in the reaction $n \rightleftharpoons p^{+}+e^{-}+\bar{\nu}$, for the coefficients we have $\nu_{n}=-\nu_{p}=-\nu_{e}=-\nu_{\nu}=1$.

We shall consider infinitesimal variations of the Gibbs potential $\left[G \equiv G\left(p, T, N_{i}\right)\right]$ with respect to the number of particles $\left(N_{i}\right)$, at constant temperature $T$ and pressure $p$ :

$$
d G=\sum_{i}\left(\frac{\partial G}{\partial N_{i}}\right)_{T, p, N_{j \neq i}} d N_{i}
$$

At chemical equilibrium, the Gibbs energy obeys the condition

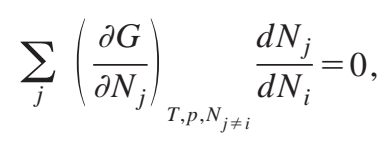

with the ratio $d N_{j} / d N_{i}$ determined by the corresponding chemical reactions. Accordingly, if an element $i$ suffers a variation $\bar{\nu}_{i}$, the remaining elements will suffer a variation $\left(\bar{\nu}_{i} / \nu_{i}\right) \nu_{j}$ to maintain the stoichiometry of the reaction. As a 
result, we have $d N_{j} / d N_{i}=\nu_{j} / \nu_{i}$, and we can write the condition of chemical equilibrium as

$$
\sum_{i} \nu_{i} \mu_{i}=0
$$

where the chemical potential of element $i, \mu_{i}$, is defined as

$$
\mu_{i} \equiv\left(\frac{\partial G}{\partial N_{i}}\right)_{T, p, N_{i \neq j}} .
$$

In this way we observe that the chemical potentials obey the symbolic equation (A1), with the substitution of $A_{i}$ by $\mu_{i}$.

In general, if a chemical reaction respects given conservation laws, the number of independent chemical potentials is equal to the number of these laws. In the following we consider two conservation laws; the electric charge and the baryon number. In this case we can express these laws, for a chemical reaction, as

$$
\sum_{i}^{N} \nu_{i} q_{e i}=0 \text { and } \sum_{i}^{N} \nu_{i} q_{b i}=0,
$$

where $q_{e i}$ and $q_{b i}$ denote the electric and baryon charges of element $i$, respectively. As in this case we have $N$ variables and two equations, we are able to express only two coefficients $\nu_{i}$ in terms of the remaining $N-2$, which will be independent:

$$
\begin{aligned}
& \nu_{1} q_{e 1}+\nu_{2} q_{e 2}=-\sum_{i \neq 1,2}^{N} \nu_{i} q_{e i}, \\
& \nu_{1} q_{b 1}+\nu_{2} q_{b 2}=-\sum_{i \neq 1,2}^{N} \nu_{i} q_{b i} .
\end{aligned}
$$

As an example we consider element 1 , the neutron and element 2 , the electron. We then have $q_{b 1}=-q_{e 2}=1, q_{b 2}$ $=q_{e 1}=0$, and the above equations become

$$
\begin{gathered}
\nu_{n}=-\sum_{i \neq n, e}^{N} \nu_{i} q_{b i}, \\
\nu_{e}=\sum_{i \neq n, e}^{N} \nu_{i} q_{e i} .
\end{gathered}
$$

Replacing Eqs. (A9) in Eq. (A4), we find

$$
\sum_{i \neq n, e}^{N} \nu_{i} \mu_{i}=\sum_{i \neq n, e}^{N}\left(\mu_{n} q_{b i}\right) \nu_{i}-\sum_{i \neq n, e}^{N}\left(\mu_{e} q_{e i}\right) \nu_{i}
$$

Since $\nu_{i}$ are independent, the equality of this equation will be verified only if the coefficients are equal. From this expression,

$$
\mu_{i}=q_{b i} \mu_{n}-q_{e i} \mu_{e}
$$

[1] A. Hewish, S. J. Bell, J. D. H. Pikington, P. F. Scott, and R. A. Collins, Nature (London) 217, 709 (1968).

[2] W. Baade and F. Zwicky, Phys. Rev. 45, 138 (1934).

[3] S. L. Shapiro and S. A. Teukolsky, Black Holes, White Dwarfs and Neutron Stars (Wiley, New York, 1983).

[4] J. D. Walecka, Advances in Nuclear Physics (Plenum, New York, 1986), Vol. 16.

[5] N. K. Glendenning, Astrophys. J. 293, 470 (1985).

[6] J. Boguta and A. R. Bodmer, Nucl. Phys. A292, 413 (1977).

[7] J. Zimanyi and S. A. Moszkowski, Phys. Rev. C 42, 1416 (1990).

[8] A. Delfino, C. T. Coelho, and M. Malheiro, Phys. Rev. C 51, 2188 (1995); Phys. Lett. B 345, 361 (1995).

[9] A. Delfino, M. Chiapparini, M. Malheiro, L. V. Belvedere, and A. O. Gattone, Z. Phys. A 355, 145 (1996).

[10] M. Chiapparini, A. Delfino, M. Malheiro, and A. Gattone, Z. Phys. A 357, 47 (1997).

[11] A. R. Taurines, MSc dissertation, Universidade Federal do Rio Grande do Sul, Porto Alegre, 1999 (unpublished).

[12] N. K. Glendenning, Compact Stars (Springer-Verlag, New York, 1997).

[13] S. A. Moszkowski, Phys. Rev. D 9, 1613 (1974).

[14] B. K. Harrison, K. S. Thorne, M. Wakano, and J. Wheeler, Gravitation Theory and Gravitational Collapse (Chicago University Press, Chicago, 1965).

[15] J. W. Negele and D. Vautherin, Nucl. Phys. A207, 298 (1973).

[16] R. C. Tolman, Phys. Rev. 55, 364 (1939).
[17] J. R. Oppenheimer and G. M. Volkoff, Phys. Rev. 55, 374 (1939).

[18] B. M. Waldhauser, J. A. Maruhn, H. Stöcker, and W. Greiner, Phys. Rev. C 38, 1003 (1988).

[19] N. K. Glendenning, F. Weber, and S. A. Moszkowski, Phys. Rev. C 45, 844 (1992).

[20] M. Barranco et al., Phys. Rev. C 44, 178 (1991).

[21] A. Delfino, J. Dey, M. Dey, and M. Malheiro, Phys. Lett. B 363, 17 (1995); Z. Phys. C 51, 2188 (1999).

[22] Z. Qian, H. Song, and R. Su, Phys. Rev. C 48, 154 (1993).

[23] M. Malheiro, A. Delfino, and C. T. Coelho, Phys. Rev. C 58, 426 (1998).

[24] W. Koepf, M. M. Sharma, and P. Ring, Nucl. Phys. A533, 95 (1992).

[25] A. Bhattacharyya and S. K. Ghosh, Int. J. Mod. Phys. E 7, 495 (1998).

[26] R. J. Furnstahl, B. D. Serot, and H.-B. Tang, Nucl. Phys. A598, 539 (1996).

[27] P. Lévai, B. Lukács, B. Waldhauser, and J. Zimányi, Phys. Lett. 117B, 5 (1986).

[28] R. Knorren, M. Prakash, and P. J. Ellis, Phys. Rev. C 52, 3470 (1995).

[29] J. Schaffner and I. N. Mishustin, Phys. Rev. C 53, 1416 (1996).

[30] I. N. Mishustin, L. M. Satarov, J. Schaffner, H. Stöcker, and W. Greiner, J. Phys. G 19, 1303 (1993).

[31] L. Landau and E. M. Lifshitz, Physique Statistique (Mir, Moscow, 1967). 